\title{
Developments of the Relativistic Bohm-Poisson Equation and Dark Energy ${ }^{\star}$
}

\author{
Carlos Castro Perelman \\ Center for Theoretical Studies of Physical Systems, Clark Atlanta University, Atlanta, GA, 30314, United States \\ Email: perelmanc@hotmail.com
}

\begin{abstract}
The nonlinear and novel Bohm-Poisson-Schrödinger equation proposed by us is studied further. It has solutions leading to repulsive gravitational behavior. An exact analytical expression for the observed vacuum energy density is obtained. Further results are provided which include two possible extensions of the Bohm-Poisson equation to the full relativistic regime. Two specific solutions to the novel Relativistic Bohm-Poisson equation (associated to a real scalar field) are provided encoding the repulsive nature of dark energy. One solution leads to an exact cancellation of the cosmological constant, but an expanding decelerating cosmos; while the other solution leads to an exponential accelerated cosmos consistent with a de Sitter phase, and whose extremely small cosmological constant is $\Lambda=\frac{3}{R_{H}^{2}}$, consistent with current observations. We conclude with some final remarks about Weyl's geometry.
\end{abstract}

Keywords: Bohm's Potential, Cosmology, Dark Energy.

\section{Introduction}

In physical cosmology and astronomy, dark energy is an unknown form of energy which is hypothesized to permeate all of space, tending to accelerate the expansion of the universe [1] . Assuming that the standard model of cosmology is correct, the best current measurements indicate that dark energy contributes 68.3 percent of the total energy in the present-day observable universe. The mass-energy of dark matter and ordinary (baryonic) matter contribute 26.8 and 4.9 percent respectively, and other components such as neutrinos and photons contribute a very small amount. The density of dark energy much less than the density of ordinary matter or dark matter within galaxies. However, it dominates the mass-energy of the universe because it is uniform across space [1]. Two proposed forms for dark energy are the cosmological constant, [2] representing a constant energy density filling space homogeneously, and scalar fields such as quintessence or moduli, dynamic quantities whose energy density can vary in time and space.

The nature of dark energy is more hypothetical than that of dark matter, and many things about the nature of dark energy remain matters of speculation [1]. Dark energy is thought to be very homogeneous, not very dense and is not known to interact through any of the fundamental forces other than gravity. In the models based on the Friedmann-Lemaitre-Robertson-Walker (FLRW) metric, it can be shown that a strong constant negative pressure in all the universe causes an acceleration in universe expansion if the universe is already expanding, or a deceleration in universe contraction if the universe is already contracting. This accelerating expansion effect is sometimes labeled "gravitational repulsion".

A major outstanding problem is that quantum field theories predict a huge cosmological constant, more than 100 orders of magnitude too large. This would need to be almost, but not exactly, cancelled by an equally large term of the opposite sign. Some supersymmetric theories require a cosmological constant that is exactly zero, which does not help because supersymmetry must be broken. Nonetheless, the cosmological constant is the most economical solution to the problem of cosmic acceleration. Thus, the current standard model of cosmology, the Lambda-CDM (cold dark matter) model, includes the cosmological constant as an essential feature [1].

The evidence for dark energy is heavily dependent on the theory of general relativity. Therefore, it is conceivable that a modification to general relativity also eliminates the need for dark energy. There are very many such theories, and research is ongoing [3], [4], [9], [12], [16]. Some authors believe that the

\footnotetext{
* This paper is dedicated to the loving memory of Francis Kamber
} 
measurement of the speed of gravity with the gravitational wave event GW170817 ruled out many modified gravity theories as alternative explanation to dark energy [1]. However, only a perfect knowledge of the motion of the LIGO mirrors and/or of the interferometer response function due to new future detections of gravitational waves will permit one to determine if general relativity is the definitive theory of gravity or if it needs some weak modification. This will happen if the sensitivity of the current gravitational wave interferometers and/or of advanced projects will improve in the future ${ }^{2}$. This issue has been recently clarified, for example, in [8].

In quintessence models of dark energy, the observed acceleration of the scale factor is caused by the potential energy of a dynamical field, referred to as quintessence field. Quintessence differs from the cosmological constant in that it can vary in space and time. In order for it not to clump and form structure like matter, the field must be very light so that it has a large Compton wavelength. This class of theories attempts to come up with an all-encompassing theory of both dark matter and dark energy as a single phenomenon that modifies the laws of gravity at various scales. This could for example treat dark energy and dark matter as different facets of the same unknown substance, a "dark fluid" [6], or postulate that cold dark matter decays into dark energy.

The Newton-Schrödinger equation has had a long history since the 1950's [18], [20]. It is the name given to the system coupling the Schrödinger equation to the Poisson equation. In the case of a single particle, this coupling is effected as follows: for the potential energy term in the Schrödinger equation take the gravitational potential energy determined by the Poisson equation from a matter density proportional to the probability density obtained from the wave-function. For a single particle of mass $m$ the coupled system of equations leads to the nonlinear and nonlocal Newton-Schrödinger integro-differential equation

$$
i \hbar \frac{\partial \Psi(\boldsymbol{r}, t)}{\partial t}=-\frac{\hbar^{2}}{2 m} \nabla^{2} \Psi(\boldsymbol{r}, t)+V(\boldsymbol{r}, t) \Psi(\boldsymbol{r}, t)-\left(G m^{2} \int \frac{\left|\Psi\left(\boldsymbol{r}^{\prime}, t\right)\right|^{2}}{\left|\boldsymbol{r}-\boldsymbol{r}^{\prime}\right|} d^{3} r^{\prime}\right) \Psi(\boldsymbol{r}, t)
$$

Exact solutions to the stationary spherically symmetric Newton-Schrödinger equation were found in terms of integrals involving generalized Gaussians [33]. The energy eigenvalues are also obtained in terms of these integrals which agree with the numerical results in the literature.

Bohm's quantum potential $V_{Q}=-\frac{\hbar^{2}}{2 m}\left(\nabla^{2} \sqrt{\rho} / \sqrt{\rho}\right)$ was shown to be proportional to the Weyl scalar spatial curvature produced by an ensemble density of paths associated with one, and only one particle, as shown in [25]. The constant of proportionality is $-\frac{\hbar^{2}}{2 m}$. It can be generalized to the relativistic case. This geometrization process of quantum mechanics allowed to derive the Schrödinger, Klein-Gordon [25] and Dirac equations [29]. Most recently, a related geometrization of quantum mechanics was proposed [32] that describes the time evolution of particles as geodesic lines in a curved space, whose curvature is induced by the quantum potential. This formulation allows therefore the incorporation of all quantum effects into the geometry of space-time, as it is the case for gravitation in the general relativity.

Based on these results we proposed in [33] the following novel ${ }^{3}$ and nonlinear quantum-like BohmPoisson equation for static solutions $\rho=\rho(\boldsymbol{r})$

$$
\nabla^{2} V_{Q}=4 \pi G m \rho \Rightarrow-\frac{\hbar^{2}}{2 m} \nabla^{2}\left(\frac{\nabla^{2} \sqrt{\rho}}{\sqrt{\rho}}\right)=4 \pi G m \rho
$$

such that one could replace the nonlinear Newton-Schrödinger equation for the above non-linear quantumlike Bohm-Poisson equation (1.2) where the fundamental quantity is no longer the wave-function $\Psi$ (complex-valued in general) but the real-valued probability density $\rho=\Psi^{*} \Psi$. The logic behind eq-(1.2) is based on the idea that the laws of physics should themselves determine the distribution of matter. This is going one step further from General Relativity where a given distribution of matter determines the geometry. Eq-(1.2) is based on Bohm's quantum potential

$$
V_{Q} \equiv-\frac{\hbar^{2}}{2 m} \frac{\nabla^{2} \sqrt{\rho}}{\sqrt{\rho}}
$$

\footnotetext{
${ }^{2}$ We thank the referee for pointing this out to us

3 To our knowledge eq-(1.2) proposed by us has not appeared before
} 
If, in addition to the Bohm-Poisson equation (1.2), one were to add the Schrödinger equation for the complex-valued wave-function $\Psi \equiv \sqrt{\rho} e^{i S / \hbar}$, one can obtain consistent solutions, which avoids having an overdetermined system of equations, when the external potential is itself a function of $\rho$ as we shall show in section 2.1. Therefore in this work we will be focusing on the Bohm-Poisson-Schrödinger system of equations instead of the Newton-Schrödinger equation (1.1).

This work is organized as follows. In section $\mathbf{2 . 1}$ we construct particular solutions to the BohmPoisson-Schrödinger equation in the spherically symmetric case. Using these solutions, in $\mathbf{2 . 2}$ we show how to obtain an exact expression for the currently observed (extremely small) vacuum energy density. In section 3.1 we review the derivation of the Klein-Gordon equation based on the relativistic version of Bohm's potential, the Hamilton-Jacobi equation and continuity equation. In section $\mathbf{3 . 2}$ we posit the novel Relativistic Bohm-Poisson equation within the context of a simple cosmological model involving a real scalar field. Two specific solutions to the Relativistic Bohm-Poisson equation are provided encoding the repulsive nature of dark energy and associated with the scalar field. One solution leads to an exact cancellation of the cosmological constant, but an expanding decelerating cosmos; while the other solution leads to an exponential accelerated cosmos consistent with a de Sitter phase, and whose extremely small cosmological constant is $\Lambda=\frac{3}{R_{H}^{2}}$, consistent with current observations. We conclude with some final remarks about Weyl's geometry.

\section{The Vacuum Energy and Bohm-Poisson-Schrödinger Equation}

\subsection{The Bohm-Poisson-Schrödinger Equation}

We may include the Schrödinger equation, in addition to the Bohm-Poisson equation (1.2), to find what is the potential $V$ which reproduces the positive definite probability density $\rho=\Psi^{*} \Psi$ and which is related to the matter density $\tilde{\rho}_{m}=m \rho$ solution of the Bohm-Poisson equation (1.2).

It is straightforward to verify that a spherically symmetric solution to eq-(2) in a $3 D$ spatially flat background ${ }^{4}$

$$
-\frac{\hbar^{2}}{2 m} \nabla^{2}\left(\frac{\nabla^{2} \sqrt{\rho_{m}}}{\sqrt{\rho_{m}}}\right)=4 \pi G m \rho_{m}, \quad \nabla^{2} f(r) \equiv r^{-2} \partial_{r}\left(r^{2} \partial_{r} f(r)\right)
$$

is given by

$$
\rho_{m}(r)=\frac{A}{r^{4}}, \quad A=-\frac{\hbar^{2}}{2 \pi G m^{2}}<0
$$

At first glance, since $\rho_{m}(r) \leq 0$, one would be inclined to dismiss such solution as being unphysical. However, one may notice that the Bohm-Poisson's (BP) equation (2.1) is invariant under $\rho_{m} \rightarrow-\rho_{m}$, and $G \rightarrow-G$. Consequently $-\rho_{m} \geq 0$ is a valid positive-definite solution to a BP equation associated to a negative gravitational coupling $-G<0$, and which is tantamount to repulsive gravity.

It remains to prove next that the external potential $V(r)$ appearing in the spherically symmetric Schrödinger equation is indeed repulsive. This does not mean, however, that $V(r)$ will just be the Newtonian potential with a change of sign. Because we have replaced the Newtonian potential for the Bohm potential in the BP equation (2.1) one should not expect this trivial change of sign to happen.

As shown by David Bohm, the Schrödinger equation for the complex valued wave function $\Psi \equiv$ $\sqrt{\rho(\boldsymbol{x}, t)} e^{i S(\boldsymbol{x}, t) / \hbar}$ is equivalent to the coupled pair of equations

$$
\begin{gathered}
-\frac{\partial S}{\partial t}=\frac{p^{2}}{2 m}+V_{Q}+V=\frac{(\nabla S)^{2}}{2 m}-\frac{\hbar^{2}}{2 m} \frac{\nabla^{2} \sqrt{\rho}}{\sqrt{\rho}}+V \\
\frac{\partial \rho}{\partial t}+\nabla\left(\rho \frac{\nabla S}{m}\right)=0
\end{gathered}
$$

The first equation is the Hamilton-Jacobi equation corresponding to an external potential $V$ and including Bohm's quantum potential $V_{Q}$; the second equation is the continuity equation. The momentum (not to be confused with pressure) is $\boldsymbol{p}=\nabla S$. Inserting the positive-definite spherically symmetric static solutions

\footnotetext{
${ }^{4}$ For the time being we shall not discuss solutions in curved backgrounds
} 
$\left|\rho_{m}\right| / m=\rho=|A| / m r^{4}$ of the Bohm-Poisson equation into the above eqs-(2.3) it leads to a coupled system of differential equations which determine the potential $V(r)$ and the action (phase) $S(r, t)=S(r)-E t$.

After some lengthy but straightforward algebra, the solutions to the Bohm-Poisson-Schrödinger equation in the spherically symmetric case are

$$
\begin{gathered}
\rho(r)=\frac{\left|\rho_{m}\right|}{m}=\frac{|A|}{m r^{4}}=\frac{1}{2 \pi G m^{3} r^{4}}, V(r)=E-\left(E-V_{o}\right)\left(\frac{r}{r_{o}}\right)^{4}+\frac{\hbar^{2}}{m r^{2}} \\
V_{Q}=-\frac{\hbar^{2}}{2 m} \frac{\nabla^{2} \sqrt{\rho}}{\sqrt{\rho}}=-\frac{\hbar^{2}}{m r^{2}} \\
S(r, t)=S(r)-E t, \quad S(r)=\sqrt{2 m\left(E-V_{o}\right)} \frac{r^{3}}{3 r_{o}^{2}}+S_{o}, E>V_{o}
\end{gathered}
$$

One can verify that the spherically symmetric wave function $\Psi(r, t) \equiv \sqrt{\rho(r)} e^{i S(r, t) / \hbar}$ built from the expressions in eqs-(2.4-2.6) is a stationary solution of the Schrödinger equation

$$
i \hbar\left(\frac{\partial \Psi(r, t)}{\partial t}\right)=-\frac{\hbar^{2}}{2 m} \frac{1}{r^{2}} \partial_{r}\left(r^{2} \partial_{r} \Psi(r, t)\right)+V(r) \Psi(r, t)
$$

with

$$
\Psi(r, t) \equiv \sqrt{\rho(r)} e^{i S(r, t) / \hbar}=\left(\sqrt{\rho(r)} e^{i S(r) / \hbar}\right) e^{-i E t / \hbar}=\Psi(r) e^{-i E t / \hbar}
$$

As expected, the external potential $V(r)$ cannot be arbitrary but is itself determined in terms of $\rho$. It can be rewritten as

$$
V(\rho(r))=c_{o}+\frac{c_{1}}{\rho(r)}+c_{2} \sqrt{\rho(r)}
$$

where $c_{o}, c_{1}, c_{2}$ are numerical coefficients given in terms of $E, V_{o}, r_{o}$. By inspection of eq-(2.4) we can see that such potential leads to a repulsive force $F=-\nabla V>0$, for $r>0$, when $E>V_{o}$. Therefore, the Bohm-Poisson-Schrödinger equation admits solutions encoding a repulsive gravity consistent with having replaced $G$ for $-G$. In the next section we shall find cosmological solutions mimicking dark energy.

The explicit presence of $\hbar^{2}$ in the expression for the external potential $V(r)$ in eq- $(2.4)$ is not very common in the Quantum Mechanical problems that we are familiar with. However, as emphasized by Klauder in his monograph [34], the principal purpose of the Enhanced Quantization program is to describe and apply a new way to quantize classical systems, which in turn, lead to classical enhanced Hamiltonians that explicitly contain nonvanishing $\hbar$ terms. A typical example of this is the introduction of Bohm's quantum potential into the classical Hamilton-Jacobi equations. This was the reason why a $\hbar$-dependent term appears in the external potential $V(r)$.

\subsection{The Vacuum Energy Density}

In the previous subsection we exploited the fact that the Bohm-Poisson's (BP) equation is invariant under $\rho_{m} \rightarrow-\rho_{m}$, and $G \rightarrow-G$, and such that $-\rho_{m} \geq 0$ is a positive-definite solution to the BP equation associated to a negative gravitational coupling $-G<0$, and which is tantamount to repulsive gravity.

In this subsection we shall explore another possibility which does not rely in changing signs but instead in adding the vacuum energy density contribution $\rho_{o}$ to the negative-definite density solution (2.2) of the BP equation $\rho_{m}$, and leading to a large region where $\rho+\rho_{o} \geq 0$.

Despite that the BP equation is nonrelativistic we can still borrow this line of reasoning (adding a constant $\rho_{o}$ to $\rho$ ) by noticing that the Einstein field equations (in spherical coordinates), when the stress energy tensor has the diagonal form, ${ }^{5}$

$$
T_{\mu \nu}=\left(-g_{t t} \rho, g_{r r} p_{(r)}, g_{\theta \theta} p_{(\theta)}, g_{\phi \phi} p_{(\phi)}\right)
$$

5 A perfect fluid in thermodynamic equilibrium in Minkowski space, for example, has in Cartesian coordinates and signature $(-,+,+,+)$ the following stress energy tensor $T_{\mu \nu}=(\rho, p, p, p), T_{\mu}^{\nu}=(-\rho, p, p, p)$ 
are given by

$$
\begin{gathered}
R_{t t}-\frac{1}{2} g_{t t} R+\Lambda g_{t t}=-8 \pi G g_{t t} \rho \\
R_{r r}-\frac{1}{2} g_{r r} R+\Lambda g_{r r}=8 \pi G g_{r r} p_{(r)}, \ldots
\end{gathered}
$$

in this case one can absorb the cosmological constant into a redefinition of $\rho$, by simply rewriting

$$
\tilde{\rho} \equiv \rho+\frac{\Lambda}{8 \pi G}=\rho+\rho_{o}
$$

leading then to field equation without the cosmological constant, but rewritten in terms of $\tilde{\rho}$ as follows

$$
R_{t t}-\frac{1}{2} g_{t t} R=-8 \pi G g_{t t} \tilde{\rho}
$$

Similarly one can do the same with the other components of the field equations by absorbing the cosmological constant into a redefinition of $p_{(r)}, \cdots$, by simply rewriting

$$
\tilde{p}_{(r)} \equiv p_{(r)}-\frac{\Lambda}{8 \pi G}=p_{(r)}-\rho_{o}, \ldots
$$

from eqs- $(2.12,2.13 \mathrm{~b})$ one infers that

$$
\tilde{\rho}+\tilde{p}_{(r)}=\rho+p_{(r)}, \tilde{\rho}+\tilde{p}_{(\theta)}=\rho+p_{(\theta)}, \ldots
$$

In the case of an isotropic fluid $p=p_{(r)}=p_{(\theta)}=p_{(\phi)}$, the dark energy equation of state $\tilde{\rho}+\tilde{p}=\rho+p=0$ remains invariant.

Therefore, borrowing this shifting procedure from the relativistic Einstein field equations to the nonrelativistic case, it gives the following expression for $\tilde{\rho}$ in terms of the above solution $(2.2) \rho_{m}$ of the nonrelativistic BP equation

$$
\tilde{\rho}=\rho_{m}+\rho_{o}=\frac{A}{r^{4}}+\rho_{0}, \quad A=-\frac{\hbar^{2}}{2 \pi G m^{2}}
$$

and consequently, when $\Lambda>0$, one can now concentrate on the domain of values of $r$ where $\tilde{\rho}(r)>0$. And, in doing so, it will permit us to show that the value of $\rho_{0}$ can be made to coincide exactly with the (extremely small) observed vacuum energy density, by simply introducing an ultraviolet length scale $l$ that is very close to the Planck scale, and infrared length scale $L$ equal to Hubble scale $R_{H}$.

A rigorous procedure would require a covariant (relativistic) extension of the BP equation. For signature $(-,+,+,+)$, it may be defined in terms of the D'Alambertian operator, and a proper mass density $\Omega(\boldsymbol{r}, t)$ of physical dimensions $(\text { length })^{-5}$, such that $m=\int \Omega(\boldsymbol{r}, t) \sqrt{|g|} d^{4} x$, as follows

$$
-\square\left(\frac{\square \sqrt{\Omega(\boldsymbol{r}, t)}}{\sqrt{\Omega(\boldsymbol{r}, t)}}\right)=4 \pi G m \Omega(\boldsymbol{r}, t), \quad \square \equiv \frac{1}{\sqrt{|g|}} \partial_{\mu}\left(\sqrt{|g|} g^{\mu \nu} \partial_{\nu}\right), \quad \hbar=c=1
$$

However, for the sake of the arguments and the discussion that follows we shall go ahead and proceed with eq-(2.14). Another relativistic (field theory) extension of the BP equations will be the subject of the next section.

Focusing for now on the static spherically symmetric solutions (2.2) of the BP equation, let us choose the ultraviolet scale $l$ to coincide with the node (zero) of $\tilde{\rho}(r)$ given by eq- $(2.14)$ such that

$$
\tilde{\rho}(r=l)=-\frac{\hbar^{2}}{2 \pi G m^{2}} \frac{1}{l^{4}}+\rho_{o}=0 \Rightarrow \rho_{o}=\frac{\hbar^{2}}{2 \pi G m^{2}} \frac{1}{l^{4}}
$$

The domain of physical values of $r$ must be $r \geq l$ in order to ensure a positive-definite density $\tilde{\rho}(r) \geq 0$. One could include all the values of $r$ from 0 to $\infty$. The density diverges at $r=0$, while the integral $\int_{0}^{\infty} \tilde{\rho}(r) 4 \pi r^{2} d r=\infty-\infty$. The $+\infty$ contribution stems from the region $r \geq l$, while the $-\infty$ contribution stems from the region $r<l$. Therefore one needs to introduce a suitable and judicious regularization involving an ultraviolet and infrared scale. 
In natural units of $\hbar=c=1$, after introducing the ultraviolet scale $l$ and infrared scale $L=R_{H}$ in the normalization condition (otherwise the mass would diverge) it yields the integral

$$
m=\int_{l}^{R_{H}} \tilde{\rho}(r) 4 \pi r^{2} d r=\int_{l}^{R_{H}}\left(\frac{A}{r^{4}}+\rho_{0}\right) 4 \pi r^{2} d r=\int_{l}^{R_{H}}\left(-\frac{1}{2 \pi G m^{2}} \frac{1}{r^{4}}+\rho_{0}\right) 4 \pi r^{2} d r
$$

In conventional QM, the plane wave solutions $\Psi=e^{i \boldsymbol{k} \cdot \boldsymbol{r}}$ are not square integrable. Nevertheless we bypass this problem after introducing an infrared cutoff by putting the free particle in a box of finite volume. Similarly, we follow this regularization procedure in eq-(2.17). Upon performing the integral in eq- $(2.17)$, and plugging in the value of $\rho_{0}$ derived from eq-(2.16), with the provision that when $R_{H}>l$ the dominant contribution to the integral stems solely from $\rho_{o}$, one ends up with the following relationship

$$
\frac{4 \pi R_{H}^{3}}{3} \rho_{o}=\frac{4 \pi R_{H}^{3}}{3} \frac{1}{2 \pi G m^{2} l^{4}}=m \Rightarrow m^{3}=\frac{2}{3} \frac{R_{H}^{3}}{G l^{4}}
$$

solving for $m$ one gets

$$
m=\left(\frac{2}{3 G l^{4}}\right)^{1 / 3} R_{H}
$$

One can verify that when the ultraviolet scale $l$ is chosen to be very close to the Planck scale, and given by

$$
l^{4}=\frac{4}{3} L_{P}^{4} \Rightarrow l=\left(\frac{4}{3}\right)^{1 / 4} L_{p}=1.0745 L_{p}
$$

then upon inserting the values for $m$ and $l$ obtained in eqs- $(2.19,2.20)$ into the expression for $\rho_{o}$ derived in eq-(2.16), after setting $L_{p}^{2}=2 G,{ }^{6}$ it gives in natural units of $\hbar=c=1$

$$
\rho_{o}=\frac{1}{2 \pi G m^{2}} \frac{1}{l^{4}}=\frac{1}{2 \pi G}\left(\frac{3 G l^{4}}{2}\right)^{2 / 3} \frac{1}{R_{H}^{2} l^{4}}=\frac{3}{8 \pi G} \frac{L_{p}^{4}}{R_{H}^{2} L_{p}^{4}}=\frac{3}{8 \pi G R_{H}^{2}}
$$

which is precisely equal to the observed vacuum energy density $\rho=(\Lambda / 8 \pi G)$ associated with a cosmological constant $\Lambda=\left(3 / R_{H}^{2}\right)$ and corresponding to a de Sitter expanding universe whose throat size is the Hubble radius $R_{H}$ ( $=c / H_{o}, H_{o}$ is today's value of the Hubble parameter).

The physical reason behind the choice of the ultraviolet scale $l$ in eq- $(2.20)$ is based on re-interpreting $\rho_{o}$ as the uniform energy (mass) density inside a black hole region of Schwarzschild radius $R=2 G m$

$$
\rho_{b h}=\frac{m}{(4 \pi / 3) R^{3}}=\frac{3}{8 \pi G R^{2}}, \quad L_{P}^{2}=2 G, \quad \hbar=c=1
$$

In the regime $R=2 G m>>l$, when the dominant contribution to the integral (2.17) stems from the $\rho_{o}$ term, we may equate the expression for $\rho_{o}$ in eq- $(2.16)$ to $\rho_{b h}$ in eq- $(2.22)$ giving

$$
\frac{1}{2 \pi G m^{2} l^{4}}=\frac{1}{2 \pi l^{4}} \frac{(2 G)^{2}}{G R^{2}}=\frac{1}{2 \pi l^{4}} \frac{L_{p}^{4}}{G R^{2}}=\frac{3}{8 \pi G R^{2}} \Rightarrow l=\left(\frac{4}{3}\right)^{1 / 4} L_{p}, \quad \hbar=c=1
$$

and leading once again to the value of $l=1.0745 L_{p}$ in eq- $(2.20)$. Therefore, when $R=2 G m>>l$, the value of $l$ is always very close to the Planck scale, and independent of $R=2 \mathrm{Gm}$, because the scale $R$ has decoupled in eq-(2.23).

In this way, one can effectively view the observable universe as a "black-hole" whose Hubble radius $R_{H}$ encloses a mass $M_{U}$ given by $2 G M_{U}=R_{H}$. From eq-(2.22) it follows that when $R=R_{H}$, the black hole density $\rho_{b h}=\rho_{o}=\rho_{o b s}$ coincides with the observed vacuum energy density. It is well known that inside the black hole horizon region the roles of $t$ and $r$ are exchanged due to the switch in the signature of the $g_{t t}, g_{r r}$ metric components. Cosmological solutions based on this $t \leftrightarrow r$ exchange were provided by the Kantowski-Sachs metric. For references on Black-Hole Cosmology see [21].

\footnotetext{
${ }^{6}$ Some authors absorb the factor of 2 inside the definition of $L_{p}$, we define the Planck scale such that the Compton wavelength coincides with the Schwarzschild radius
} 
To conclude this section, our interpretation of these results of being able to express the vacuum energy density $\rho_{o}$ in terms of an ultraviolet (Planck) and infrared scale (Hubble scale) stems from the inherent non-locality of Bohm's formulation of QM. Such non-locality is reflected in this ultraviolet/infrared entanglement. For another perspective of this small/large scale entanglement within the context of Scale Relativiy Theory see [50].

\section{Dark Energy and the Relativistic Bohm-Poisson Equation}

\subsection{Derivation of the Klein-Gordon Equation}

The relativistic version of the Bohm potential for a scalar field is [25], [29]

$$
V_{Q}=\hbar^{2} \frac{\square\left(\sqrt{\phi^{*}(\boldsymbol{r}, t) \phi(\boldsymbol{r}, t)}\right)}{\sqrt{\phi^{*}(\boldsymbol{r}, t) \phi(\boldsymbol{r}, t)}}
$$

Note that $V_{Q}$ has now physical units of $(\text { mass })^{2}$ instead of mass. Given the signature $(+,-,-,-)$ the relativistic analog of the Hamilton-Jacobi equation eq- $(2.3 \mathrm{a})$ is

$$
\left(\partial_{\mu} S\right)^{2}=m^{2}+V_{Q}=m^{2}+\hbar^{2} \frac{\square\left(\sqrt{\phi^{*}(\boldsymbol{r}, t) \phi(\boldsymbol{r}, t)}\right)}{\sqrt{\phi^{*}(\boldsymbol{r}, t) \phi(\boldsymbol{r}, t)}}
$$

the four-current is

$$
J_{\mu}=i\left(\phi^{*}(\boldsymbol{r}, t) \partial_{\mu} \phi(\boldsymbol{r}, t)-\phi(\boldsymbol{r}, t) \partial_{\mu} \phi^{*}(\boldsymbol{r}, t)\right)
$$

and obeys the conservation law (continuity equation)

$$
\partial_{\mu} J^{\mu}=0
$$

related to the conservation of a Noether charge $Q=\int J_{\mu} d \Sigma^{\mu}$ that is given by the flux of the current $J_{\mu}$ through a spatial 3-surface $\Sigma^{\mu}$. $Q$ counts the number of scalar particles minus the number of anti-particles flowing through the 3-spatial surface. In QFT (relativistic QM) the scalar field $\phi$ is no longer a wave function, hence it is not related to a probability amplitude as such.

Writing the complex scalar field in the polar form

$$
\phi \equiv\|\phi(\boldsymbol{r}, t)\| e^{i S(\boldsymbol{r}, t) / \hbar}=\sqrt{\phi^{*}(\boldsymbol{r}, t) \phi(\boldsymbol{r}, t)} e^{i S(\boldsymbol{r}, t) / \hbar}
$$

allows to solve for $S=-\frac{i \hbar}{2} \ln \left(\frac{\phi}{\phi^{*}}\right)$. Then a lengthy but straightforward algebra leads to the Klein-Gordon equation 7

$$
\left(\hbar^{2} \square+m^{2}\right) \phi(\boldsymbol{r}, t)=0, \quad\left(\hbar^{2} \square+m^{2}\right) \phi^{*}(\boldsymbol{r}, t)=0
$$

It was noticed long ago by [25] that the relativistic version of Bohm's potential $V_{Q}(3.1)$ is proportional to the Weyl scalar curvature $R_{W}$ in flat spacetime backgrounds when Weyl's gauge field of dilatations is $A_{\mu} \sim \partial_{\mu} \ln \left(\phi^{*} \phi\right)$. Because $A_{\mu}$ is pure gauge (total derivative) the Weyl's field strength $F_{\mu \nu}=\partial_{\mu} A_{\nu}-$ $\partial_{\nu} A_{\mu}=0$, which implies that the rate of the ticking of clocks will be independent of their paths taken from point $A$ to $B$. Consequently, atomic clocks arriving on earth via different trajectories will tick at the same rate (same spectral lines). In this fashion one can avoid Einstein's criticism of Weyl's gravity.

A conformally covariant equation ${ }^{8}$ equation in curved backgrounds in $4 D$ with a curvature scalar coupling, can also be obtained via Bohm's quantum potential [25], [29]

$$
\left(\hbar^{2} g^{\mu \nu} D_{\mu} D_{\nu}+m^{2}+\frac{R_{W}}{6}\right) \phi(\boldsymbol{r}, t)=\left(\hbar^{2} g^{\mu \nu} \nabla_{\mu} \nabla_{\nu}+m^{2}+\frac{R}{6}\right) \phi(\boldsymbol{r}, t)=0
$$

where $D_{\mu}=\nabla_{\mu}+A_{\mu}$ is the Weyl covariant derivative and $R_{W}$ is the Weyl scalar curvature. The "conformal" mass $m$ parameter is posited to scale under Weyl scalings with a Weyl weight of -1 . The

\footnotetext{
7 Using a different signature $(-,+,+,+)$ requires changing the signs in the right hand side of $(3.2)$ and it leads to the Klein-Goldon equation with a sign change in the $m^{2}$ term

${ }^{8}$ The homogeneous differential equation is also conformally invariant
} 
weight of $g^{\mu \nu}$ and $R_{W}$ is -2 , while the weight of $\phi$ is -1 . Due to key factor of $\frac{1}{6}$ (that varies with the spacetime dimension) in the Weyl scalar curvature $R_{W}$, the field $A_{\mu}$ decouples entirely from the left hand side of the equation leading to the right hand side expressed solely in terms of the Riemannian scalar curvature $R$ and covariant derivatives $\nabla_{\mu}$ based on the Christoffel connection.

A Weyl-gauge invariant proof of the spin-statistics theorem, and solving the Quantum nonlocality enigma by Weyl's Conformal Geometry can be found in more recent work by [27]. The coupling to the Electromagnetic field via the prescription $p_{\mu} \rightarrow p_{\mu}-e A_{\mu}$ leads to a modified Klein-Gordon equation by simply replacing $\square$ with $\left(\partial_{\mu}-i e A_{\mu}\right)\left(\partial^{\mu}-i e A^{\mu}\right)$.

To conlude this brief review of the Klein-Gordon equation, one should add that the deep question of whether or not Bohmian mechanics can be be made relativistic was studied in [31]. In relativistic space-time, Bohmian theories can be formulated by introducing a privileged foliation of space-time. The introduction of such a foliation - as extra absolute space-time structure - would seem to imply a clear violation of Lorentz invariance, and thus a conflict with fundamental relativity. The authors [31] considered the possibility that, instead of positing it as extra structure, the required foliation could be covariantly determined by the wave function. This allowed for the formulation of Bohmian theories that seem to qualify as fundamentally Lorentz invariant. They concluded with some discussion of whether or not they might also qualify as fundamentally relativistic.

\subsection{Dynamical Dark Energy and the Relativistic Bohm-Poisson Equation}

Having reviewed briefly how to derive the Klein-Gordon equation based on the relativistic version of Bohm's potential we proceed with the cosmological applications. Given the Lorentzian signature $(-,+,+,+)$, let us begin with the action in a curved background with a cosmological constant

$$
S=\int d^{4} x \sqrt{-g}\left(\frac{(R-2 \Lambda)}{16 \pi G}-\frac{g^{\mu \nu}}{2}\left(\partial_{\mu} \phi\right)\left(\partial_{\nu} \phi\right)-V(\phi)\right)
$$

and associated with a canonical real scalar field $\phi$ with a potential $V(\phi)$. The FLRW metric is

$$
d s^{2}=-(d t)^{2}+a^{2}(t)\left(\frac{(d r)^{2}}{1-k r^{2}}+r^{2}(d \Omega)^{2}\right), \quad k=1,0 ;-1
$$

$k$ is the spatial scalar curvature parameter with units of $(\text { length })^{-2}$. More precisely, $k \rightarrow \frac{k}{l^{2}}$, where the scale $l$ has been set to unity.

Inserting this metric into the Einstein field equations yield in $c=1$ units

$$
\begin{gathered}
a^{-2}\left(\frac{d a}{d t}\right)^{2}+\frac{k}{a^{2}}-\frac{\Lambda}{3}=\frac{8 \pi G}{3} \rho \\
2 a^{-1}\left(\frac{d^{2} a}{d t^{2}}\right)+a^{-2}\left(\frac{d a}{d t}\right)^{2}+\frac{k}{a^{2}}-\Lambda=-8 \pi G p
\end{gathered}
$$

From now one we shall abbreviate the temporal derivatives by $\partial_{t} a=\dot{a} ; \partial_{t}^{2} a=\ddot{a}, \cdots$ when appropriate.

The latter pair of equations is equivalent to the following pair of equations

$$
\begin{gathered}
\dot{\rho}=-3 \frac{\dot{a}}{a}(\rho+p) \\
\frac{\ddot{a}}{a}=-\frac{4 \pi G}{3}(\rho+3 p)+\frac{\Lambda}{3}
\end{gathered}
$$

Eq-(3.11a) is just the local energy-momentum conservation law $\nabla^{\mu} T_{\mu \nu}=0$ [49], and eq-(3.11b) reflects the fact that a positive density and pressure cause a deceleration, while a positive $\Lambda$ causes an acceleration in the expansion of the universe.

One must supplement eqs-(3.10) (or eqs-(3.11)) with the equations of motion for the $\phi$ field $\square \phi\left(x^{\mu}\right)=\frac{d V(\phi)}{d \phi}$. In the special case $\phi=\phi(t)$, it becomes

$$
-a^{-3}(t) \partial_{t}\left(a^{3}(t) \partial_{t} \phi(t)\right)=\frac{d V(\phi)}{d \phi}
$$


The real scalar field $\phi=\phi(t)$ is assumed to behave as a perfect fluid whose density and pressure are [4]

$$
\rho=\frac{1}{2}\left(\frac{d \phi}{d t}\right)^{2}+V(\phi), \quad p=\frac{1}{2}\left(\frac{d \phi}{d t}\right)^{2}-V(\phi)
$$

Because eq-(3.11a) is just the local energy-momentum conservation law $\nabla^{\mu} T_{\mu \nu}=0$ which is satisfied for all potentials $V(\phi)$, the system of 3 equations $(3.11,3.11 \mathrm{~b}, 3.12)$ is not sufficient to determine the potential $V(\phi)$. Thus one must specify a priori the expression for the potential. When the cosmological constant is set to zero $\Lambda=0$, by suitably choosing the potential, it is still possible to reproduce the cosmic expansion at late times. There is a plethora of different models which are possible for different potentials. For example, one potential is the Ratras-Peeble potential $V(\phi)=M^{4+n} \phi^{-n}$, with $n>0$ [4]. Another potential is the Starobinksy potential $V=\Lambda^{4}\left(1-e^{-\alpha \phi}\right)^{2}[5]$, with $\alpha=\left(\sqrt{\frac{2}{3}} / M_{P}\right), M_{P}$ is the Planck mass.

The difference now is that we shall not set $\Lambda$ to zero, and we shall also derive the functional form of the potential function $V(\phi)$ by adding the relativistic analog of the Bohm-Poisson equation, instead of being an a priori suitably chosen function. Previously we showed how to derive the form of the repulsive potential $V(r)$ from the solutions to the Bohm-Poisson-Schrödinger equation. We are going to follow a similar procedure for the relativistic case.

To simplify matters, let us take a real-valued field $\phi=\phi^{*}$. The relativistic, field theory analog of the Bohm-Poisson equation in $D$ dimensions, that we propose in this work is defined by the following equation

$$
\left(\frac{\hbar}{m}\right)^{2} \square\left(\frac{\square \phi(\boldsymbol{r}, t)}{\phi(\boldsymbol{r}, t)}\right)=4 \pi G g^{\mu \nu} T_{\mu \nu}, \quad \square \equiv \frac{1}{\sqrt{|g|}} \partial_{\mu}\left(\sqrt{|g|} g^{\mu \nu} \partial_{\nu}\right), \quad c=1
$$

where the trace of the stress energy tensor $T=g^{\mu \nu} T_{\mu \nu}$ appears in the right hand side. Both sides of eq-(3.14) are scalars as they should. The gravitational coupling constant in $D$-dimensions is related to the Planck scale in $D$-dimensions by $G_{D} \sim L_{P}^{D-2}$. Eq-(3.14) has the same physical units as the cosmological constant $\Lambda$.

In passing we should mention that of the many articles surveyed in the literature pertaining the role of Bohm's quantum potential and cosmology, [36], [43], [44] we did not find any related to the novel Bohm-Poisson equation proposed in this work. ${ }^{9}$. The authors [43], for instance, have shown that replacing classical geodesics with quantal (Bohmian) trajectories gives rise to a quantum corrected Raychaudhuri equation (QRE). They derived the second order Friedmann equations from the QRE, and showed that this also contains a couple of quantum correction terms, the first of which can be interpreted as cosmological constant (and gives a correct estimate of its observed value), while the second as a radiation term in the early universe, which gets rid of the big-bang singularity and predicts an infinite age of our universe. The model of "dark energy without dark energy" based on the sub-quantum potential associated with the CMB particles by [47] also differs from the work presented here.

From eqs-(3.13) one obtains the trace of the stress energy tensor $T$

$$
\begin{gathered}
T=-\rho+3 p=(\dot{\phi})^{2}-4 V(\phi)= \\
-\frac{3}{8 \pi G}\left(\left(\frac{\dot{a}}{a}\right)^{2}+\frac{k}{a^{2}}-\frac{\Lambda}{3}\right)-\frac{3}{8 \pi G}\left(2\left(\frac{\ddot{a}}{a}\right)+\left(\frac{\dot{a}}{a}\right)^{2}+\frac{k}{a^{2}}-\Lambda\right)
\end{gathered}
$$

Inserting $T$ into eq-(3.14) actually leads to two separate equations

$$
\left(\frac{\hbar}{m}\right)^{2} \square\left(\frac{\square \phi(\boldsymbol{r}, t)}{\phi(\boldsymbol{r}, t)}\right)=4 \pi G\left((\dot{\phi})^{2}-4 V(\phi)\right)
$$

and

$$
\left(\frac{\hbar}{m}\right)^{2} \square\left(\frac{\square \phi(\boldsymbol{r}, t)}{\phi(\boldsymbol{r}, t)}\right)=-3\left(\frac{\ddot{a}}{a}+\left(\frac{\dot{a}}{a}\right)^{2}+\frac{k}{a^{2}}-\frac{2 \Lambda}{3}\right)
$$

\footnotetext{
${ }^{9}$ A Google Scholar search provided the response "Bohm-Poisson equation and cosmological constant did not match any articles"
} 
For complex-valued fields, one simply replaces $\phi$ for $\sqrt{\phi^{*} \phi}$ in the left hand side of (3.14), whereas for the right hand side one must evaluate the stress energy tensor, and its trace corresponding to complex scalar fields. To simplify matters we shall focus on real-valued scalar fields. As usual, one must supplement eqs- $(3.16,3.17)$ with the equations of motion for the scalar $\phi$ field. When $\phi=\phi(t)$, it becomes

$$
\square \phi(t)=-a^{-3}(t) \partial_{t}\left(a^{3}(t) \partial_{t} \phi(t)\right)=\frac{d V(\phi)}{d \phi}
$$

To sum up, one has a coupled system of 3 differential equations $(3.16,3.17,3.18)$ to solve for $a(t), \phi(t)$, and $V(\phi)$. Things simplify considerably in the massless case $m=0$. Because then one may bring the $m$ factor into the right-hand side of eqs- $(3.16,3.17)$, and then set $m=0$, leading now to one single equation

$$
m=0 \Rightarrow \square\left(\frac{\square \phi(\boldsymbol{r}, t)}{\phi(\boldsymbol{r}, t)}\right)=a^{-3}(t) \partial_{t}\left(a^{3}(t) \partial_{t}\left[a^{-3}(t) \phi^{-1}(t) \partial_{t}\left(a^{3}(t) \partial_{t} \phi(t)\right)\right]\right)=0
$$

Note that despite we set $m=0$ this does not force the trace $T$ to be zero because the action (3.8) is not conformally invariant. In this massless case, one must still include eq-(3.15), which in conjunction with eqs-(3.18, 3.19), furnishes a simpler coupled system of 3 differential equations to solve for $a(t), \phi(t)$, and $V(\phi)$.

We are going to find a simple, but not trivial solution, to eqs- $(3.15,3.18,3.19)$ when the spatial curvature parameter $k=0$. A solution can simply be found by requiring

$$
\square \phi(t)=-a^{-3}(t) \partial_{t}\left(a^{3}(t) \partial_{t} \phi(t)\right)=-(3 H \dot{\phi}+\ddot{\phi})=\frac{d V(\phi)}{d \phi}=0
$$

where the Hubble function is defined by $H(t) \equiv \frac{\dot{a}}{a}$.

After some lengthy but straightforward algebra, one finds that the solutions to eqs- $(3.18,3.19,3.20)$ are

$$
\begin{gathered}
\phi(t)=\frac{1}{2 \sqrt{3 \pi G}} \ln \left(\frac{t}{t_{o}}\right), \quad a(t)=a_{o}\left(\frac{t}{t_{o}}\right)^{\frac{1}{3}} \\
H(t)=\frac{1}{3} t^{-1}, \quad V=V_{o}=-\frac{\Lambda}{8 \pi G} \\
\rho(t)=\frac{1}{24 \pi G}\left(t^{-2}-3 \Lambda\right), \quad p(t)=\frac{1}{24 \pi G}\left(t^{-2}+3 \Lambda\right)
\end{gathered}
$$

The upshot of these solutions (3.21) obtained when $m=k=0$, is :

(i) At late times, $t \rightarrow \infty, \rho=-p=-\frac{\Lambda}{8 \pi G}$ which corresponds to the dark energy equation of state. Choosing the sign of $\Lambda$ to be $\Lambda<0$ yields $\rho>0$ and $p<0$ (negative pressure).

(ii) If one sets $t_{o}$ to coincide with the present value of the Hubble time $t_{H}=\left(H_{o}\right)^{-1}$ (equal to $R_{H}$ in $c=1$ units), at the present time $t=t_{o}$, the value of the scalar field $\phi$ vanishes but not its derivative $\dot{\phi}\left(t=t_{H}\right)=\frac{t_{H}^{-1}}{2 \sqrt{3 \pi G}}$. Because the logarithmic function has a slow growth, despite that the values of the scalar field will remain very small (almost vanishing) for times $t \sim t_{H}$, there is a non-vanishing energy density permeating all of space. For instance, the present day value of the kinetic energy density $\frac{1}{2}(\dot{\phi})^{2}$ is $\frac{1}{24 \pi G R_{H}^{2}}$, and which is quite close to the observed vacuum energy density $\frac{3}{8 \pi G R_{H}^{2}}$.

(iii) Inserting the constant value of the potential $V=V_{o}=-\frac{\Lambda}{8 \pi G}$ back into the classical action (3.8) it will cancel exactly the cosmological constant term, irrespective of the value and sign of $\Lambda$. If one wishes to identify $\frac{\Lambda}{8 \pi G}$ with the enormous vacuum energy density due to the zero-point fluctuations of the fields associated with the Standard Model, it will be cancelled exactly by the $V_{o}$ contribution in the action (3.8). Therefore, the relativistic Bohm-Poisson equation associated with the massless real scalar field, when the spatial curvature parameter is chosen to be $k=0$, provides a natural and simple mechanism to cancel the cosmological constant in the action (3.8) without invoking supersymmetry. A QFT with no zero-point energy has been recently advanced by [35].

There is a caveat because, rigorously speaking, one would have to include the contribution of the Standard Model fields into the action in order to study the dynamics of all the fields. In turn, this would 
affect the functional form of the new solutions, and an exact cancellation of $\Lambda$ might no longer occur. Nevertheless it is encouraging that a simple action like (3.8) permits an exact cancellation of $\Lambda$.

(iv) In the $m=0$ case, $\hbar$ decouples from all the equations giving a "classical" appearance (flavor) to all the equations. However eq-(3.19) is intrinsically Quantum Mechanical because it is based on the relativistic version of Bohm's Quantum potential. When $m \neq 0$, $\hbar$ will no longer decouple from the solutions to the equations.

(v) The scaling factor $a(t)$ (3.21) obeys $\dot{a}>0$, and $\ddot{a}<0$, hence it corresponds to an expanding, but decelerating, universe. Inserting the solutions (3.21) one can verify that the $\Lambda$ terms cancel out exactly in eq-(3.11b) yielding a decelerating $\operatorname{cosmos} \frac{\ddot{a}}{a}=-\frac{2}{9} t^{-2}<0$.

(vi) The scalar spacetime curvature associated with the scaling factor $a(t)=a_{o}\left(\frac{t}{t_{o}}\right)^{\frac{1}{3}}$, when $k=0$, is

$$
R=6\left(\frac{\ddot{a}}{a}+\left(\frac{\dot{a}}{a}\right)^{2}\right)=-\frac{2}{3} t^{-2}
$$

and it asymptotically vanishes as $t \rightarrow \infty$. Similarly, the kinetic terms in the action (3.8) vanish in that limit. One may also notice that the action (3.28) vanishes on-shell for all values of $t$ ( $\left.\operatorname{since} g^{t t}=-1\right)$. The magnitude of $R$ at the present-day value of the Hubble time (Hubble scale) $t_{H}=R_{H}$ is $|R|=\frac{2}{3 R_{H}^{2}}$ which is quite close to the (extremely small) observed value of $\Lambda=\frac{3}{R_{H}^{2}}$.

(vii) $R$ and $\phi$ blow up at $t=0$, consistent with the Big-Bang singularity.

(viii) When $k=0$, one may verify by simple inspection that the solutions to eqs- $(3.15,3.18,3.19)$, given by eqs-(3.21a, 3.21b, 3.21c), also solve eqs-(3.10a, 3.10b, 3.18). This is a sign of consistency.

Finally, we proceed to find other solutions which correspond to an exponentially expanding cosmos like de Sitter space. Upon inserting the ansatz $a=e^{H_{o} t}$ into eqs-(3.15, 3.18, 3.19), and solving for the function $\Theta \equiv \frac{\square \phi(t)}{\phi(t)}$ which obeys eq-(3.19) $\square \Theta=0$, gives

$$
\Theta \equiv \frac{\square \phi(t)}{\phi}=A e^{-3 H_{o} t}, \quad A \neq 0
$$

which in turn yields the following set of equations

$$
\begin{gathered}
\ddot{\phi}-2 A e^{-3 H_{o} t} \phi=0 \\
3 H_{o} \dot{\phi}+\frac{3}{2} \ddot{\phi}=0 \\
(\dot{\phi})^{2}-4 V=-\frac{3}{2 \pi G}\left(H_{o}^{2}-\frac{\Lambda}{3}\right)
\end{gathered}
$$

after using

$$
\Theta \equiv \frac{\square \phi(t)}{\phi(t)}=\phi^{-1} \frac{d V}{d \phi}=\phi^{-1} \frac{\dot{V}}{\dot{\phi}}=\frac{1}{2} \frac{\ddot{\phi}}{\phi}=A e^{-3 H_{o} t}
$$

and obtained from differentiating eq-(3.26). There are no nontrivial solutions to the pair of equations $(3.24,3.25)$. The solutions to $(3.24)$ are given in terms of Bessel functions; whereas the solutions to (3.25) are given in terms of simple exponentials. Therefore the solutions to eqs- $(3.24,3.25,3.26)$ are

$$
\phi(t)=0, \quad V=V_{o}=\frac{3}{8 \pi G}\left(H_{o}^{2}-\frac{\Lambda}{3}\right)
$$

Plugging $V_{o}$ back into the classical action (3.8) leads to an exact cancellation of $\Lambda$, irrespective of its value and sign, leaving only the remaining term $-\frac{3 H_{o}^{2}}{8 \pi G}$, and which coincides with the contribution of the vacuum energy density (critical energy density). There is no problem with the minus sign in front, because it will be offset by another minus sign in the definition of the stress energy tensor $T_{\mu \nu}=-\frac{2}{\sqrt{|g|}} \frac{\delta\left(\sqrt{|g|} \mathcal{L}_{m}\right)}{\delta g^{\mu \nu}}$ giving a positive value in the right hand side of the field equations.

Whereas, if $V_{o}$ in eq-(3.28) is set to zero $V_{o}=0 \Rightarrow \Lambda=3 H_{o}^{2}=\frac{3}{R_{H}^{2}}$, it then yields the observed extremely small value of the cosmological constant. Either way, whether or not we set $V_{o}$ to zero, leads to 
the same value of the vacuum energy density. To sum up, in this case the classical action (3.8) reduces to a pure gravitational action with a very small cosmological constant $\Lambda=\frac{3}{R_{H}^{2}}$ corresponding to an exponential expanding cosmos in a de Sitter phase, as it is currently observed.

Let us compare the solutions (3.28) (when $a(t)=e^{H_{o} t}$ ) with the solutions to the 3 equations (3.10a, $3.10 \mathrm{~b}, 3.12)$ when $a(t)=e^{H_{o} t}$, and $k=0$. These solutions are $\phi=\phi_{o}=$ constant and $V_{o}=\frac{3}{8 \pi G}\left(H_{o}^{2}-\frac{\Lambda}{3}\right)$. By choosing $\phi_{o}=0$ one then recovers the solutions (3.28) once again. This is a sign of consistency.

In the most general case, in $4 D$ there are 10 Einstein field equations for the 10 independent metric components $g_{\mu \nu}$. There is one equation of motion $\square \phi=\frac{d V}{d \phi}$ for the $\phi\left(x^{\mu}\right)$ field giving 11 equations for a total of 12 unknown functions $\left\{g_{\mu \nu}, \phi, V(\phi)\right\}$. Adding the relativistic Bohm-equation (3.14) will then bring the total number of equations to 12 which matches now the number of 12 unknown functions.

There are myriads of other solutions for the $m=0, k=0$ case. Also when $k \neq 0$, and/or $m \neq 0$. Given $k=1,0,-1$, and $m=0, m \neq 0$, there are 6 cases to consider within the FLRW metrics, and each one of them provides many different families of solutions. The solutions in the most general case are not easy to obtain. The main result of this work is that we have been able to find solutions in two cases with clear physical relevance in Cosmology. In particular, the second solution yields the very small value $\frac{3}{R_{H}^{2}}$ of $\Lambda$ when $V_{o}=0$.

To conclude, in the most general case, the relativistic Bohm-Poisson equation is a prescription to solve (determine) the potential $V(\phi)$ in a system of 12 coupled nonlinear partial differential equations corresponding to 12 functions $\left\{g_{\mu \nu} ; \phi ; V(\phi)\right\}$ in four dimensions. It can be generalized to other dimensions as well. This method relies on the co-existence of the classical and quantum world (see also [34]) since it combines classical equations with the relativistic Bohm-Poisson equation (quantum like), like the Newton-Schrödinger equation which involves a combination of both classical (Newton) and quantum (Schrödinger) equations.

One can extend the action (3.8) to one involving conformal gravity based on Weyl's geometry. The results in [25] led to a key relationship between the Weyl gauge field $A_{\mu}$ and the scalar $\phi$ of the form

$$
D_{\mu} \phi=0 \Rightarrow A_{\mu} \sim-\partial_{\mu} \ln \left(\frac{\phi}{\phi_{o}}\right) \Rightarrow \phi \sim \phi_{o} e^{-\int A_{\mu} d x^{\mu}}
$$

where $D_{\mu}$ is the Weyl covariant derivative. Therefore, the Weyl-covariantly-constant scalar field $\phi$ (which is a source of dark energy) can be identified with minus the exponential of the line integral ${ }^{10}$ of the Weyl gauge field $A_{\mu}$ of dilatations. Since $A_{\mu}$ is pure gauge, locally it can be gauged to zero, but not globally since there may be topological obstructions in doing so. For this reason it is warranted to explore what role non-trivial topologies might play in Cosmology. If $A_{\mu}$ can be gauged to zero globally, then the scalar $\phi=\phi_{o}$ can be set (gauged) to a constant.

A more recent proposal that advocates the fall of dark matter may be found in [17]. Scale invariance is assumed in the empty regions of space. The Weyl gauge field $A_{\mu}$ contributes to modifications of the Christoffel connection leading then to repulsive corrections to the geodesic equations. Another approach to solve these cosmological puzzles based on the scaling properties of fractals can be found in Nottale's Scale Relativiy Theory [50]. Conformal gravity has also been proposed as a solution of the problems with dark matter and dark energy by [15]. The role of Weyl geometry warrants further investigation. Another project that needs to be studied is the issue of instabilities. Because the Relativistic Bohm Poisson equation has higher derivatives than two this question has to be studied further.

Acknowledgments. We are indebted to M. Bowers for assistance; to J.F. Gonzales for the reference about the work of [47], and to the referee for bringing to our attention the work in [8].

\section{References}

1. "Dark Energy" http://en.wikipedia.org/wiki/Dark_energy

2. S. Carroll, "The cosmological constant". Living Reviews in Relativity 4 (2001) 1.

${ }_{10}$ The line integral can be taken from a path starting at $\infty$ and ending at the point $x^{\mu}$ where the real scalar $\phi$ field is being evaluated 
3. M. Sami and R. Myrzakulov, "Late time cosmic acceleration: ABCD of dark energy and modified theories of gravity". International Journal of Modern Physics D 251630031 (2016).

4. A. Joyce, L. Lombriser, F. Schmidt, "Dark Energy vs. Modified Gravity". Annual Review of Nuclear and Particle Science 66 (2016) 1-29.

5. A. Starobinsky, "Spectrum Of Relict Gravitational Radiation And The Early State Of The Universe". Journal of Experimental and Theoretical Physics Letters. 30 (1979) 682.

6. A. Arbey, "Is it possible to consider Dark Energy and Dark Matter as a same and unique Dark Fluid? http://arxiv.org/abs/astro-ph/0506732"

7. A. Arbey, "Dark Fluid: a complex scalar field to unify dark energy and dark matter http://arxiv.org/abs/astro$\mathrm{ph} / 0601274 "$

8. C. Corda, "The future of gravitational theories in the era of the gravitational wave astronomy" International Journal of Modern Physics D 27, No. 5 (2018) 1850060.

9. S. Capozziello and M. De Laurentis "The dark matter problem from $f(R)$ gravity viewpoint" , Annalen der Physik 524, 9-10 (2012) 545-578.

10. S. Capozziello and M. De Laurentis, "Extended Theories of Gravity" Phys. Rep. 509 4-5 (2011) 167-321.

11. S. Capozziello, P. Jovanovic, V. Borka Jovanovic and D. Borka"Addressing the missing matter problem in galaxies through a new fundamental gravitational radius" JCAP 06 (2017) 044.

12. M. Milgrom, "A modification of the Newtonian dynamics as a possible alternative to the hidden mass hypothesis". Astrophysical Journal. 270 (1983) 365-370.

13. P. Kroupa, M. Pawlowski and M. Milgrom (2012). "The failures of the standard model of cosmology require a new paradigm". International Journal of Modern Physics D 21 (14) (2012) 1230003.

14. J. Bekenstein, "Relativistic gravitation theory for the modified Newtonian dynamics paradigm", Physical Review D 70 (8) (2004) 083509.

15. P. Mannheim, "Alternatives to Dark Matter and Dark Energy", Prog. Part. Nucl. Phys. 56 (2) (2005) 340.

16. B.G. Sidharth and A. Das, "Anomaly of Dark Matter" arXiv : 1609.04246

17. A. Maeder, "Dynamical effects of the scale invariance of the empty space: The fall of dark matter ?" arXiv : 1711.07740.

18. S.I. Pekar, "Untersuchungen uber die Elektronentheorie der Kristalle" Akademie Verlag, Berlin, 1954, pp-29-34.

19. R. Ruffini and S. Bonazzola, "Systems of self-gravitating particles in general relativity and the concept of an equation of state", Phys. Rev. 187 (1969) 1767

20. M. Bahrami, A.Grossardt, S. Donadi, and A. Bassi, "The Schrödinger-Newton equation and its foundations" New J. Phys. 16 (2014) 115007.

21. R. Pathria, "The Universe as a Black Hole". Nature. 240 (5379): 298-299.

22. I. Goody, "Chinese universes". Physics Today. 25 (1972) 7.

23. P. Landsberg, "Mass Scales and the Cosmological Coincidences". Annalen der Physik. 496 (2) (1984) 88-92.

24. Black Hole Cosmology, http://en.wikipedia.org/wiki/Black_hole_cosmology

25. E. Santamato, "Geometric derivation of the Schrödinger equation from classical mechanics in curved Weyl spaces", Phys. Rev. D 29, (1984) 216.

26. E. Santamato, "Statistical interpretation of the Klein-Gordon equation in terms of the space-time Weyl curvature", Journal of Mathematical Physics 25, 2477 (1984).

27. E. Santamato and F. De Martini. "A Weyl-gauge invariant proof of the Spin-Statistics Theorem" arXiv : 1604.06253.

28. E. Santamato and F. De Martini. "Solving the Quantum Nonlocality Enigma by Weyl's Conformal Geometry Geometrodynamics" arXiv : 1203.0033 .

29. C. Castro, "Nonlinear Quantum Mechanics as the Weyl Geometry of a Classical Statistical Ensemble ". Found. Physics Letters, vol.4, no.1 (1991) 81.

30. C. Castro, "On Weyl Geometry, Random Processes and Geometric Quantum Mechanics ". Foundations of Physics. vol 22, no.4 (1992) 569- 615.

31. D. Durr, S. Goldstein, T. Norsen, W. Struyve, Nino Zangh?, "Can Bohmian mechanics be made relativistic?" arXiv : 1307.1714 .

32. I. Tavernelli, "On the Geometrization of Quantum Mechanics" Annals of Physics 371 (2016) 239.

33. C. Castro, "Exact Solutions of the Newton-Schroedinger Equation, Infinite Derivative Gravity and Schwarzschild Atoms" Physics and Astronomy International Journal 1, issue 4 (2017) 1-8

34. J. Klauder, Enhanced Quantization: Particles, Fields and Gravity, World Scientific, 2015.

35. J. Klauder, "Quantum Field Theory with No Zero-point energy", arXiv : 1803.05823.

36. A. Faraggi, "The Equivalence Postulate of Quantum Mechanics, Dark Energy and The Intrinsic Curvature of Elementary Particles", arXiv : 1204.3185.

37. A. Shojai and F. Shojai, "Constraints Algebra and Equations of Motion in Bohmian Interpretation of Quantum Gravity", arXiv : gr-qc/0311076. 
38. D. Fiscaletti, "Bohm's Quantum Potential and the Geometry of Space", Quantum Matter 2, 1 (2013) 45.

39. E. Squires, "A quantum solution to a cosmological mystery", Phys. Letts A 162, 1 (1992) 35.

40. J. Vink, "The quantum potential interpretation of quantum mechanics due to Bohm is applied to the Wheeler-De Witt equation for minisuperspace" Nuc. Phys. B 369, 3 (1992) 707.

41. T. Horiguchi, Mod. Phys. Lett. A 9, (1994).

42. J. Marto and P. Vargas Moniz, "de Broglie-Bohm FRW universes in quantum string cosmology", Phys. Rev. D 65, (2001) 023516.

43. A. Farag and S. Das, "Cosmology from quantum potential", PLB $\mathbf{7 4 1}$ (2015) 276.

44. H. Nikolic, "Time and Probability : From classical mechanics to relativistic Bohmian mechanics" arXiv : 1309.0400

45. H. Nikolic, "Cosmological constant, semiclassical gravity, and foundations of quantum mechanics" grqc/0611037.

46. H. Nikolic, "Interpretation miniatures" arXiv : 1703.08341

47. Pedro F. Gonzalez-Diaz, "Dark energy without dark energy", Phys. Rev. D 69 (2004) 103512.

48. P.F. Gonzalez-Diaz and A. Rozas-Fernandez, Phys. Lett. B 641 (2006) 134.

49. R. Wald, General Relativity (page 100, University of Chicago Press, 1984).

50. L. Nottale, "Mach's Principle, Dirac's Large Number Hypothesis and the Cosmological Constant Problem", http://www.luth.obspm.fr/luthier/nottale/arlambda.pdf (1993).

51. L. Nottale, Scale Relativity And Fractal Space-Time: A New Approach to Unifying Relativity and Quantum Mechanics (World Scientific 2011)

52. L. Nottale, Fractal Space-Time and Micro-physics (World Scientific 1993). 